(Aus dem Institut zur Erforschung der Folgeerscheinungen von Hirnverletzungen [Abteilg. des Neurologischen Instituts der Universität Frankfurt a. M.].)

\title{
Psychologische Analysen hirnpathologischer Fälle auf Grund von Untersuchungen Hirnverletzter.
}

\author{
VII. \\ Über Gesichtsfeldbefunde bei abnormer "Ermüdbarkeit" des Auges \\ [sog. ,Ringskotome" $]^{1}$ ). \\ Von \\ Adhémar Gelb und Kurt Goldstein.
}

Mit Tafel I und 2 Textabbildungen.

Mehrfach sind von ophthalmologischer Seite, ,ring-förmige Skotome' beschrieben worden - so von Inouye $\left.{ }^{2}\right),\left(\right.$ ölfflin $\left.^{3}\right), J^{6}{ }^{4}$ ), Zade ${ }^{5}$ ), Doesschate ${ }^{6}$ ) -, für die eine befriedigende Erklärung bisher nicht gefunden wurde.

Im wesentlichen half man sich mit der Annahme, es handele sich um eine hysterische Erscheinung (Inouye, Wölfflin), oder man erklärte die Entstehung der Ringskotome für unklar, stellte nur eine gewisse Analogie fest zu anderen, ringförmig auftretenden krankhaften Veränderungen dex Netzhaut. Als Ursache für sie diskutierte man dann

1) Zuerst mitgeteilt auf der Vers. süd-westd. Neurol. u. Psych. in Baden-Baden 1918. Zeitschr. f. d. ges. Neurol. u. Psychiatr., Orig., 1918.

2) Inouye, Die Sehstörungen bei Schußverletzungen der corticalen Sehsphäre. Leipzig 1909.

3) E. Wölflin, Über ein seltenes Gesichtsfeldsymptom bei Hysterie. Arch. f. Augenheilk. 65, 309ff. 1910.

4) Jess, Die Ringskotome nach Sonnenblendung. Arch. f. Augenheilk. 74. 1913. - Derselbe, Augenärztliche Kriegserfahrungen, in der ,Sammlung zwangloser Abhandlungen aus dem Gebiete der Augenheilkunde", hrsg. von $A$. Vossius, Bd. 10, Heft 3, S. 32 ff.

5) M. Zade, a) Periphere Ringskotome. Graefes Arch. f. Ophthalmol. 91, 159ff. 1916. Vgl, auch: Über Blendung im Fliegerdienst. Ber, üb. d. 40. Versammlung der ophthalmol. Gesellsch. (Heidelberg 1916), Wiesbaden 1916, S. $222 \mathrm{ff}$. sowie: Über Blendungserscheinungen im Felde. Münch. med. Wochenschr. 62, Nr. 44, S. 1514ff. 1915. - b) Periphere Ringskotome II. Graefes Arch. f. Ophthalmol. 100, 129ff. 1919. (Hier auch die ältere Literatur.) - c) Ringskotome im Telegraphendienst. Zeitschr. f. Augenheilk. 43, 681. 1920.

$\left.{ }^{6}\right)$ G. ten Doesschate, Über Gesichtsfeldstörungen bei Fliegeroffizieren. Zeitschr. f. Augenheilk. 39, 30ff. 1918. 
entweder eine Störung der Blutversorgung oder eine Schädigung der Nervenfaserschicht in der Netzhaut (cf. Zade, dieses Arch. 100, S. 134).

Charakteristisch ist, daß allen Erörterungen die stillschweigende Voraussetzung zugrunde liegt, es handele sich bei den betreffenden Gesichtsfeldbefunden tatsächlich um skotomartige Ausfälle des Gesichtsfeldes in Form eines Ringes.

Wir selbst hatten an einer Reihe von Hirnverletzten derartige Ge. sichtsfeldbefunde gewonnen. Die nähere Cntersuchung solcher Kranker führte bald zu einer eindeutigen Auffassung über das Zustandekommen der ,,ringförmigen Skotome ${ }^{\text {: }}$ : es zeigte sich, um das vorwegzunehmen, vor allem, daß es sich bei unseren Kranken jedenfalls nicht um eigentliche Skotome handelte, und es scheint uns die Vermutung nahe zu liegen, daß es sich auch bei den von anderen Autoren veröfentlichten Fällen wohl ebenso verhalten hat.

Fassen wir zunächst einiges Tateachenmaterial ins Auge. Bei einem unserer Hirnverletzten stellten wir, wenn das Prüfungsobjekt in jedem Meridian von außen bis zum Fixierpunkt geführt wurde, das in Fig. Ia und $1 \mathrm{~b}$ abgebildete Gesichtsfeld fest (vgl. Tafel I), Prüfungsobjekt: $1 \mathrm{~cm}$ Quadrat WeiB. Sämtliche anderen Gesichtsfelder sind mit dem gleichen Prüfungsobjekt aufgenommen.

Fall 1 (zu Fig. 1). J. Ka., 25 Jahre alt, Naurer.

Am 5. IX. 1917 Artilleriegeschoßverletzung am linken Schädel.

April 1918. Am Arsatz der linken Ohrmuschel etwa exbsengroße, gut verschiebliche Einschußnarbe. Auf der linken Schädelseite läßt sich röntgenologisch ein etwa $2 \mathrm{~cm}$ langer und $2 \mathrm{~cm}$ breiter Metallsplitter tief im Gehirn nachweisen, und zwar bei der Aufnahmo von vorn 3 Querfinger oberhalb des oberen Augenhöhlenrandes, bei seitlicher Aufnahme 2 Querfinger oberhalb des Cehörganges. K. klagt über starke Kopfschmerzen mit Schwindelgefühl und Herabsetzung der allgemeinen Leistungsfähigkeit. Augenbewegungen nach rechts unangenehm und otwas ersehwert. Beim Stehen mit geschlossenen Augen Fallen nach links. Beim Gehen mit geschlossenen Augen Abweichen nach links. Zeigeversuch: Vorbeizeigen nach außen und unten mit der linken Hand. Ophthalmologiseh ohno Störung.

Fig. 1 zeigt neben einer nicht anbeträohtlichen allgemeinen Gesichtsfeldein. schränkung ein ,ringjörmiges Skotom", einen Ring, der etwa parallel zur Außengrenze des Gesichtsfeldes verläuft. Seine Breite sehwankt zwischen 8 und $15^{\circ}$. Zwischen dem „Ring ${ }^{* 6}$ und der Außengrenze des Gesichtsfeldes befindet sich eine Zone, ungefähr ebenso breit wie der „Ring“ selbst, in der der Pat. das Prüfungsm objekt in anscheinend normaler Weise sieht.

Die Zone zwischen ".Ring" und Außengrenze wollen wir als ..Außenzone", das zentrale erhaltene Gebiet aber als ,innere Partie“ bezeichnen.

Der Pat. beschreibt den Eindruok, den er bei zentripetaler Objektfiuhrung längs eines beliebigen Meridians hat, gewöhnlich folgendermaßen: Das Objekt wird (an der AuBengrenzo) sichtbar, verschwindet jedoch sehr bald in einem dichten, grauweiBlichen Nebel oder wird wenigstens ganz versohlejert, um dann nach laurzer theit abermals aufzutauchen. Das wiederholt sich in ganz ähnlicher Weise in jedem Meridian.

Die zentrale Sehøchärfe (nach Snellen) sehwankt bei diesem Pat. beiderseits etwa 
zwischen $1 / \mathrm{g}$ und $1 / 5$ der normalen. Bei länger andauernder zentraler Fixation nimmt sie immer mehr ab, bis der Pat. nur noch "Nebol" vor den Augen sieht.

Die Farbenperzeption ist nur in dem fovealem Bezirk gut erhalten, aber auch da nur so lange, als die Sehschärfe eine noch relativ befriedigende bleibt. Parazentral ist der Farbensinn immer herabgesetz $t^{1}$ ).

Der Pat. klagt, daß er auch im gevöhnlichem Leben (im diffusen Tageslicht) am Rande seines Gesichtsfeldes meistens "Nebel" sieht, so daß die indirekt gesehenen Gegenstände oft ,wie hinter einem dichten Schleier" erscheinen. Der "Nebel" werde um so dichter, je schlechter das Allgemeinbefinden sei; bei einiger Anstrengung der Augen ,werde es ibberall (evtl. im ganzen Gesichtsfeld) nebelig ${ }^{\text {ci }}$.

Ganz ähnliche Gesichtsfelder konnten wir dann bei einer Reihe anderer Patienten - im ganzen bei 6 Patienten - mit verschieden lokalisierten Hirnverletzungen beobachten. Als Beispiele bringen wir die Gesichtsfelder der rechten Augen von 3 anderen Patienten (Fall 2, 3 und 4 ; vgl. die Fig. 2-4).

Fall 2 (zu Fig. 2). A. D., Musketier (Schreiner).

10. X. 1917. Artilleriegesehoßverletzung am rechten Hinterhaupt.

Februar 1918. Auf dem rechten Hinterianptsbein von der Mittellinie lateralwärts ziehend $6 \times 3 \mathrm{~cm}$ große, gegen die Unterlage schlecht verschiebliche Narbe, röntgenologisch keine sichtbare Schädelverletzung, kleine Granatsplitter in den Weichteilen des Hinterkopfes, Blutung in cerebro. - Gleichgewichtsstörungen, insbesondere Unsicherheit beim Gehen und Stehen. Symptome einer doppelseitigen zentralen Vestibularläsion mit Beteiligung des rechten Kleinhirnes. Starke Herabsetzungen der a.llgemeinen körperlichen und geistigen Loistungsfähigkeit.

$\left.\begin{array}{l}\text { R. S. } 5 / 35 \\ \text { L. S. } 5 / 25\end{array}\right\}$ Gläser bessern nicht.

Ophthalmologisch ohne Befund. Angeborene Farbenblindheit (Deuteranopie).

Fall 3 (zu Fig. 3). W. H., 22 Jahre alt, Kaufmann.

Am 16. VII. 1916 Minensplitterverletzung.

Mai 1918. An der Grenze des linken Scheitels und Hinterhauptes $10 \mathrm{~cm}$ lange, auf der Unterlage gut verschiebliche, horizontal verlaufende Narbe. Am hinteren Ende etwas druckempfindlich.

H. klagt über Kopfschmerz, Schwindelanfälle, allgemeine Utbererregbarkeit und starke Ermüdbarkeit. Keine gröberen organisehen Ausfallerscheinungen. Sehschärfe mit Korrektur: rechts $1 / 2$, links $2 / 3$, doch bei den einzelnen Untersuchungen stark schwankend. Ophthalmologisch ohne Störung.

Fall 4 (zu Fig. 4). K. J., 23 Jahre alt (Werkzeugmacher).

Am 20. IX. 1915. Schrapnellschußverletzung. Einschuß: Linkes Nasenloch.

Mai 1918. Geschoß sitzt, wie röntgenologisch nachweisbar, in der hinteren Schädelgrube.

Innere Organe o. B. Nervensystem: Rechte Pupille weiter als die linke, reagiert gut auf Licht und bei Akkomodation. Augenbewegungen nach links ersohwert und unangenehm. Geringer Nystagmus nach beiden Seiten.

Reflexe, Motilität und Sensibilität intakt.

Romberg: Fallen nach rechts hinten.

Beim Gehen mit geschlossenen Augen starkes Abweichen nach rechts. Beim Fingernasenversuch mit der rechten. Hand Vorbeizeigen nach links außen, mit der linken Hand nach rechts auken.

1) Sporiellere Fragen bezitglich des Farbensinns interessicren uns hier weiter nicht und sollen daher unerörtert bleiben. 
Beim Zeigeversuch mit der linken Hand Vorbeizeigen nach auBen und oben, mit der rechten Hand ebenfalls nach außen und oben. Das Vorbeizeigen rechts stärker als links. Bei Prüfung der Schwerempfindung fiodet sich eine starke Übersehätzung links. gefühl.

Beim Bücken starke Cyanose des Gesichtes und ausgesprochenes Schwindel-

Die ohrenärztliche Untersuchung ergab linksseitige Schwerhörigkeit and rechtsseitige Schädigung der zentralen Gleichgewichtsbahnen.

Sehschärfe: Reehts $5 / 7$ ohne Glas; $5 / 5$ mit $-0,5 \mathrm{D}$.

Links: $5 / 15$ ohne Clas; $5 / 7$ knapp mit - $0,75 \mathrm{D}$.

Ophthalmologiseh: Die temporale Hälfte der Papille lejeht blaß; am linken Ange dicht unterhalb der Macula feine Reste früherer Blutungen.

J. kJagt über häufigen Schwindel und starke Kopfschmerzen. Er sei sehr leicht orregbar, schlafe sehr schlecht, habe keinen Appotit, habe zwar versucht, etwas zn arbeiten, könne jedoch kaum etwas leisten.

Die bisher besprochenen Gesichtsfelder (Fig. 1-4) weisen ausgesprochene Übereinstimmungen auf:

1. Bei allen 4 Patienten verlaufen die Ringe etwa parallel zur AuBengrenze.

2. Die "AuBenzonen" haben überall ungefähr dieselbe Breite.

3. Die "Ringe" sind nicht an allen Stellen im selben Maße amblyopisch (in den Abbildungen durch verschieden dichte Strichelung veranschaulicht).

Eine völlige "Blindheit" im ganzen ,Ringe" kommt nur äußerst selten vor. Wenigstens werden stärkere Reize (z. B. größere Prüfungs:objekte), wenn die Patienten ausgeruht sind, an sämtlichen Stellen des „Ringes" gesehen, wenn auch evtl. sehr undeutlich und verschleiert. Freilich, ein weißes $1 \mathrm{~cm}$ Quadrat verschwindet - wenigstens in der Mitte des "Ringes" - gewöhnlich rollständig, an manchen Stellen sieht. es nur verschleiert aus, es erscheint wie ,von Nebel verhüllt".

Unterschiede dagegen zeigen sich hauptsächlich darin, daB die , Ringe" bei den verschiedenen Patienten eine verschiedene Breite haben.

Der Gedanke, daß es sich bei derartigen Gesichtsfeldbefunden um Folgen einex Läsion oder einer Erkrankung streng umaschriebener Partien des Sehorgans handelt, der Leitungsbahnen oder noch zentraler gelegener Abschnitte, erscheint natürlich von vornherein als im äußersten Maße unwahrscheinlich, denn man wird sich kaum vorstellen können, daß ein Herd die Sehbahn auf beiden Seiten so geschädigt hat - noch dazu in so vielen Fällen in übereinstimmender Weise -, daß die homo. nymen ,Skotome" beider Seiten sich zu einem ,Ring" ergänzen.

Daß es sich hier in der Tat nicht um Folgen umschriebener Läsionen handelt, ließen uns unsere weiteren Untersuchungen sehr bald erkennen. Es zeigte sich nämlich, daß die Gesichtsfelder unserer Patienten fast bei jeder neuen Perimeteruntersuchung eine andere Form und Ausdehnung aufwiesen; insbesondere ergab sich, daB die "Ringe" an verschiedenen 
Tagen ganz verschiedenen Partien der „Netzhaut"1) entsprachen; je nachdem, ob die Außengrenzen weiter nach außen oder mehr nach innen lagen, lagen auch die "Ringe" bald weiter, bald weniger weit von der maculären Zone entfernt.

Als Beispiele hierfür bringen wir Gesichtsfelder von den Fällen 1, 2 und 4, die den Aufnahmen desselben Auges der Patienten zu verschiedenen Zeiten entsprechen. (Fig. 5a-b [Fall 1], Fig. 6a-b [Fall 2], Fig. $7 \mathrm{a}-\mathrm{b}$ [Fall 4]).

Bei allen drei Patienten zeigt sich sehr deutlich, daß die Lage des „Ringes" wechselt. Mit dem Wechsel der Lage der „Ringe" wechselt auch die Außenzone ihre Lage, bleibt aber immer annähernd gleich breit.

Etwas ganz Analoges ergab sich auch bei den übrigen Patienten.

Auf Grund der Tatsache, daß die Lage der „Ringe" wechselt, können wir mit Sicherheit behaupten, daß wir es nicht mit wirklichen (positiven) Skotomen zu tun haben. Denn wären die "Ringe" wirkliche Skotome, d. h. Stellen aufgehobener oder verminderter Empfindlichkeit, die ganz bestimmten Partien des Sehapparates entsprechen, so könnten sie natürlich nicht in der dargelegten Weise ihre Lage ändern.

Die eigentümlichen Befunde weisen darauf hin, daß sie wohl durch eine Funktionsstörung allgemeiner Natur bedingt sein müssen. Sie werden verständlich, wenn man sie als Folgen einer abnorm starken Ermüdung des Sehorgans auffaßt. Bei der Ermüdung des Normalen, z. B. wenn ein Normaler (im diffusen Tageslicht) seinen Blick auf eine weiße Marke auf einem schwarzen Grunde richtet, etwa am Perimeter, und die zentrale Fixation streng innehält, tritt mit der Zeit zunehmender "Verbrauch" der "Sehsubstanz" (Hering) ein; das Auge ,ermüdet" immer mehr. Zuerst werden die periphersten und peripheren, dann die parazentralen und endlich die zentralen Partien der Netzhaut ,unterwertig“, so daß zuerst die ganz peripher gebotenen Gegenstände, z. B. ein sehr peripher gehaltenes, ruhendes Untersuchungsobjekt, dann die parazentral gebotenen Objekte und schlieBlich auch die weiße Fixationsmarke unsichtbar werden: Alles scheint sich in einen ,grauen Nebel" aufzulösen, der sich von außen mehr und mehr gegen den Fixationspunkt vorschiebt.

Die Schnelligkeit, mit welcher sich dieser Vorgang abspielt, ist je nach den Umständen verschieden; sie hängt beim Normalen von den gerade vorhandenen äußeren Prüfungsbedingungen und der speziellen physiologischen Zuständlichkeit (,Stimmung" des Auges) ab.

Wird die Fixation aufgegeben, das Auge bewegt und damit durch andere Reize erregt als bei der Fixation, so „erholt" sich das Auge.

1) Unter „Netzhaut" verstehen wir hier das, ,imnere Auge " im Simne Herings, d. h. Netzhaut, Sehnerv und die entsprechenden Hirnteile. 
Man bemerkt, daß der ,Nebel" schwindet und die Objekte wicder deutlich werden.

Auf die möglichen Erklärungen der beschriebenen Ermüdungstrscheinungen wollen wir hier nicht eingehen. Ein Verständnis dafür im großen ganzen kann man auf Grund der Anschauungen ge'winnen, die E. Hering in seiner Theorie vom Lichtsinn über das chemische Geschehen in der "Sehsubstanz" unter der Einwirkung innerer und äußerer Reize (die Dissimilations- und Assimilationsvorgänge) und insbesondere die Vorgänge bei der ,simultanen Licht. induktion" entwickelt hat ${ }^{1}$ ).

In pathologischen Fällen kann unter den geschilderten Umständen der ",Verbrauch der Sehsubstanz", die „Ermüdbarkeit" des Auges -gleichviel zunächst aus welchen Gründen - so ungewöhnlich stark sein, daß die Objekte unvergleichlich schneller undeutlich werden als beim Normalen.

Gehen wir mit diesen Grundvoraussetzungen an die Erklärung unserer Befunde heran, so wird zunächst die Klage unserer Patienten verständlich, daß sie bei anhaltender zentraler Fixation schon nach wenigen Augenblicken den Perimeterreifen und etwas später auch dic Fixationsscheibe ganz undeutlich, wie im ,Nebel", sehen. Daß diese Klage nicht übertrieben war, zeigte sich objektiv darin, daß man bei allen Patienten bei fortlaufendem Perimetrieren etwa im Sinne des Uhrzeigers schon nach etwa $1 \frac{1}{2}$, Ermüdungstouren" (Wilbrand) eine „spiralförmige", hochgradige Finengung der AuBengrenze des Ge. wichtsfeldes erzielen konnte.

Besonders deutlich zeigte sich die rasch zunehmende Einengung der Außengrenzen z. B. bei Fall 1 bei einer campimetrischen Untersuchung ${ }^{2}$ ) (Prüfungsobjekt $=$ weißes $1 \mathrm{~cm}$ Quadrat; Prüfungsabstand $=120 \mathrm{~cm}$ ), bei der das Objekt auf dem gewählten Meridian von außen bis zum Fixationspunkt durchgeführt wurde. Dabei ergaben sich für

1) Bezüglich des Näheren verweisen wir auf folgende Arbeiten von Hering: Zur Lehre vom Lichtsinn. Wien 1878, Zur Theorie der Vorgänge in der lebendigen Substanz, Lotos. Bd. 9. Prag 1888. Über Erholung und Ermiidung des Sehorgans. Lotos 3\%. - Eine übersichtliche, kurz gehaltene Darstellung der Heringschen Lehre findet der neurologisch und ophthalmologisch interessierte Leser in der Arbeit von $H$. Wilbrand, Utber die Frholungsausdehnung des Gesichtsfeldes unter normalen wad pathologischen Bedingungen" (Wiesbaden 1896), der die Heringsohe Theorie, wenn auch mitunter etwas einseitig und wohl picht immer mit Recht, zur Enklärung der verschiedensten Erscheinungen, die bei Gesichtsfeldprüfungen hervortreten, heranzieht.

2) Wir benutzen als Campimeter einen mit mattschwarzem Stoff bespannten Rahmen von $3 \times 3 \mathrm{~m}$ Größe und tragen die gewonnenen linearen MaBe auf das später (S. 395) abgedruckte Schema ein, nicht etwa die umgerechneten Winkelwerte.

Vgl. hierzu Gelb und Goldstein: Das röhrenförmige Gesichtsfeld usw. im Neurol. Centralbl. 1918. 
das rechte Auge folgende Werte (die cm bedeuten die Entfernungen der Prüfungsmarke vom Fixationspunkte):

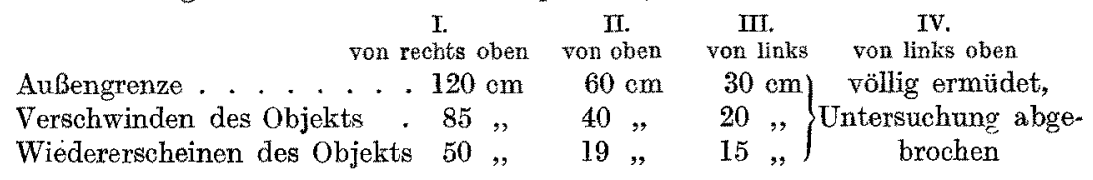

Nach 2 Minuten Pause: Von rechts im horizontalen Meridian: Außengrenze $12 \mathrm{~cm}$. Dann verschwindet das Objekt.

Die ungewöhnlich starke Ermüdbarkeit war auch die Ursache davon, daß die Außengrenzen des Gesichtsfeldes unserer Patienten so außerordentlich wenig konstant waren. (Man betrachte in dieser Hinsicht die Fig. 5, 6, 7 und vergleiche sie untereinander.) Da der Grad der Ermüdbarkeit von außerordentlich vielen äußeren und inneren Faktoren abhängt, die nicht konstant bleiben, ist fast bei jeder neuen Perimeteruntersuchung die Konstellation der betreffenden Faktoren eine andere. Ein ungewöhnlich leicht ermüdendes Auge wird, da es dem Einflusse der verschiedenen, die Frmüdung bewirkenden Faktoren in besonders hohem Maße ausgesetzt ist, bei den verschiedenen Gesichtsfeldprüfungen einen sehr verschiedenen Grad der Ermüdbarkeit aufweisen, was außerordentlich schwankende Gesichtsfeldgrenzen zur Folge haben muß.

Wie erklärt sich nùn der Hauptbefund, das Zustandekommen der „,Ringe"? Kurz gesagt: dadurch, daß das Prüfungsobjekt beim Perimetrieren von außen nach innen mit einer bestimmten Geschwindigkeit bewegt wird. Ließe man nämlich das Prüfungsobjekt an der peripheren Stelle, wo es zuerst sichtbar wurde, dauernd anf das Auge unserer Patienten einwirken, so würde es schon nach wenigen Augenblicken unsichtbar werden, da infolge der ,Ermüdung“ die Empfindlichkeit der Netzhaut gerade an den periphersten Stellen zuerst ${ }^{\top}$ erlischt. Wir bekämen also auf diese Weise einfach eine Einschränkung des Gesichtsfeldes. Bewegt man dagegen das Objekt zentripetal weiter, bevor an der ersten Stelle die Unempfindlichkeit auftritt, so verschwindet es an einer etwas zentraler gelegenen benachbarten Partie. Auf diese Weise entsteht der Schein, als wenn das Objekt erst an dieser zentraleren Partie unsichtbar wurde, während in Wirklichkeit im Moment des Unsichtbarwerdens des Objektes auch die äußerste Peripherie hochgradig amblyopisch geworden, also gar keine ,AAußenzone" vorhanden ist.

Bei weiterer Bewegung des Objektes gegen noch mehr zentral gelegene Partien wird das Objekt wieder sichtbar, weil es jetzt eine Netzhautregion trifft, die infolge ihrer größeren physiologischen Wertigkeit nicht bis zum Erlöschen der Empfindlichkeit ermüdet ist. Würde man das Objekt an dieser neuen Stelle wiederum dauernd einwirken lassen, so würde das ruhende Objekt auch hier nach kurzer Zeit verschwinden oder sehr undeutlich werden; da aber das Objekt mit gleichmäBiger Ge- 
schwindigkeit gegen den Fixationspunkt weiter bewegt wird, verschwindet es überhaup tnicht mehr (vgl. indessen später: S. 396 f.), denn bei der weiteren Bewegung trifft es immer auf Partien, die trotz der zunehmenden Ermüdung ihre Empfindlichkeit noch nicht völlig eingebüßt haben.

Die oben abgebildeten , iypischen Ringskotome" sind demnach einfach als Produkte der Untersuchungsmethode aufzufassen. Sie kommen dadurch zustande, daß das Prüfungsobjekt relativ schneller zentripetal bewegt wird, als die Frmüdung des Sehorgans von außen nach innen fortschreitet. Es handelt sich also um die Wirkung eines ganz bestimmten quantitativen VerhäItnisses dex Schnelligkeit der Objektführung zum Fortschreiten der Ermüdung.

Ist das richtig; so müssen Änderungen dieses Verhältnisses zu charakteristischen Veränderungen der typischen Ringskotome führen, und ferner müssen sich die veränderten Gesichtsfelder alle von dem Gesichtspunkte aus erklären lassen, daß die Ringskotome Effekte des erwähnten quantitativen Verhältnisses darsiellen.

Die im folgenden zu berichtende Reihe von Versuchstatsachen möge zur Verifikation unserer Annahme dienen:

1. Da die Ermüdung von außen nach innen fortschreitet, so muß, wenn das Prüfungsobjekt aus dem „Ring" nicht weiter gegen die Fixationsscheibe hin, sondern wieder rückwärts, gegen die Außengrenze geführt wird, das Gebiet, in dem die Außenzone lag, jetzt unempfindlich sein, weil die Ermüdung dieses Gebietes noch stärker sein muß als im ,Bing".

In der Tat blieb das Prüfungsobjekt unsichtbar, wenn man es aus dem „Ring“ wieder nach außen führte. Voraussetzung dafür war freilich, daß der Patient die Fixation streng innehielt, weil schon die geringste Augenbewegung innerhalb der Fixationsscheibe ein wichtiges „Erholungsmoment" bildet, so daß bei nicht gut eingehaltener Fixation das Prüfungsobjekt zuweilen wieder sichtbar werden konnte ${ }^{1}$ ).

2. Ein Fehlen der Außenzone muß sich nach unserer Annahme auch zeigen, wenn man die ganze Gesichtsfelduntersuchung von vornherein mit einer zentrifugalen Objektführung vornimmt. Während bei einer zentripetalen Objektfillhung die Außenzone - mit vereinzelten Ausnahmen (vgl. unten S. 395) - so gut wie immer da ist, fehlt sie bei zentrifugaler Objektführung, wiederum vorausgesetzt, daß die zentrale Fixation vom Patienten gut innegehalten wird. Im letzten Falle wird das Objekt mit fortschreitender Bewegung nach außen immer undeutlicher und verschwindet schließlich ganz, ohne daß es an der Peripherie wieder sichtbar wird. Dabei erklären die Kranken gewöhnlich spontan,

1) Näheres darüber in der (oben S. 392 Anm. ${ }^{1}$ ) angegebenen Schrift von Wilbrand uber die Erholungsausdehnung des Gesichtsfeldes usw. S. 15ff. 
daß sie bei zentrifugaler Objektführung ,schlechter sähen", als bei zentripetaler. Perimetrierte man z. B. Fall 2 auf der temporalen Seite im horizontalen Meridian von außen nach innen, so tauchte das Objekt bei $65^{\circ}$ zum erstenmal auf, wurde bei $52^{\circ}$ undeutlich und bei $40^{\circ}$ wieder deutlich. Bei zentrifugaler Objektführung dagegen verschwand das Prüfungsobjekt bei ca. $50^{\circ}$, im Nebel " und wurde überhaupt nicht mehr sichtbar.

Die verschiedenen Resultate bei zentripetaler und zentrifugaler Objektführung zeigten sich noch deutlicher bei einer campimetrischen Untersuchung (Prüfungsabstand $=1 \mathrm{~m}$ ). Ein Beispiel mag auch das erläutern (Text-Fig. la und $1 b$ ):

Bei zentripetaler Objektführung tauchte das Objekt auf der temporalen Seite des horizontalen Meridians bei $100 \mathrm{~cm}$ Entfernung vom

Rechtes Auge.

Zentripetale Objektführung

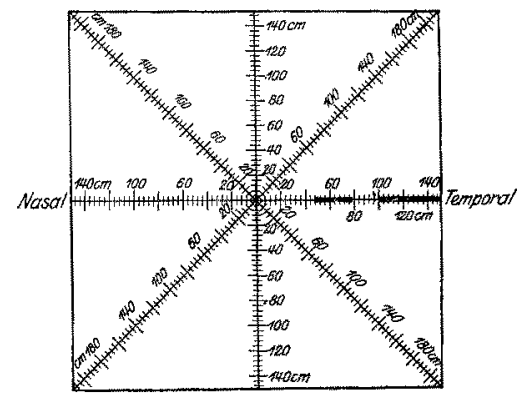

Fig. 1 a.

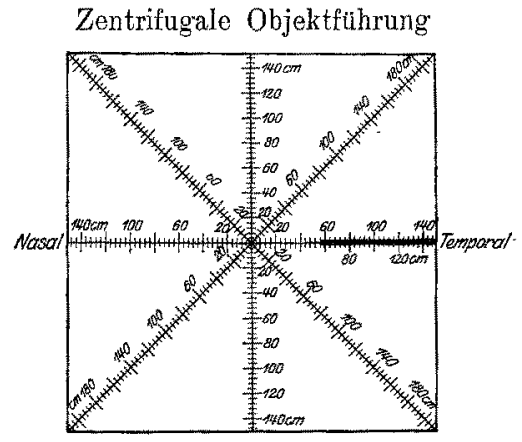

Fig. $1 \mathrm{~b}$.

Maßstab $1: 80$.

Fixationspunkt auf, verschwand bei $79 \mathrm{~cm}$ Entfernung und tauchte bei $49 \mathrm{~cm}$ wieder auf. Bei zentrifugaler Objektführung verschwand das Objekt in $56 \mathrm{~cm}$ Entfernung vom Fixationspunkt und wurde überhaupt nicht mehr gesehen. Hier zeigt sich also besonders deutlich, daß eine Partie des Gesichtsfeldes, in der der Patient bei zentripetaler Objektführung das Prüfungsobjekt sah, bei dem andern Versuchsverfahren ,blind" war.

Gelegentlich kommt es während einer Untersuchung vor, daß auch bei zentripetaler Objektführung die Außenzone gar nicht auftritt. Das Objekt erscheint in manchen Meridianen schon an den periphersten Partien des Gesichtsfeldes undeutlich, also schon dort, wo sonst die sog. Außenzone liegt, und wird erst an Stellen deutlich, wo gewöhnlich die innere Partie zu beginnen pflegt (vgl. auf der Tafel in Fig. 8 den oberen linken und in Fig. 9 den oberen rechten Quadranten. Gesichtsfelder von Fall 1.) 
Das erklärt sich aus einer abnorm großenErmüdbarkeit: Die Emptindlichkeit der äußeren Peripherie war in diesem Falle schon erloschen oder so stark herabgesetzt, daß es zu gar keinem deutlichem Sehen des Objektes kommen konnte. Das Objekt/wird erst in Regionen deutlich, in denen die Wertigkeit der Netzhaut verhältnismäßig groß ist, und auch nur dann, wenn die Objektführung schneller vor sich geht, als die Ermüdung zentralwärts fortschreitet; sonst liegt die gut empfindliche Zone nahe am Fixationspunkt. Der Einfluß der abnormen Ermüdung des Sehorgans zeigt sich auch darin, daß man einen geschlossenen gleichmäßigen „Ring", wie ihn die Fig. 1-4 auf der Tafel zeigen, nur dann erhält, wenn man dem Patienten nach der Untersuchung jedes einzelnen Meridians eine lourze Erholungs pause gönnt. Prüft man alle Meridiane nacheinander, ohne den Patienten inzwischen ausruhen zu lassen, so tritt im weiteren Verlauf der Untersuchung eine Verbreiterung des Ringes ein, die mitunter so groß sein kann, daß der ,Ring" bis zum Fixierpunkt reicht (vgl, in Fig. 9 den linken unteren Quadranten), oder ein Näherrücken der Außenzone an das Zentrum (vgl. in Fig. 9 unten). Da das Näherrücken der Außenzone gegen das Zentrum bei einer fortlaufenden Untersuchung immer gegen Ende derselben auftritt, so muB der Gesichtsfeld befund sich ändern, je nachdem, ob man, etwa an der unteren Hälfte des vertikalen Meridians anfangend, die einzelnen Meridiane nacheinander - ohne den Patienten ausruhen zu lassen - im Sinne der Uhrzeigerbewegung oder in der umgekehrten Richtung prüft. Ein deutliches Beispiel hierfür ist der Befund bei Fall 4 (vgl. Fig. 10a und 10b). Die beigefügten Zahlen längs der normalen Außengrenze bedeuten die Reihenfolge der untersuchten Meridiane.

Es ist nur eine weitere Bestätigung unserer Anschauung, daß an sog. schlechten Tagen der Patienten, d. h. bei schlechtem Allgemeinbefinden der Patienten, das Prüfungsobjekt überhaupt nur ganz am Anfang der Untersuchung (bei der Prüfung des zuerst gewählten oder der beiden ersten Meridiane) an der Außengrenze des Gesichtsfeldes gut sichtbar wird, während es im ganzen übrigen Gesichtsfelde, wenn überhaupt, so erst in der maculären Zone einigermaßen deutlich erscheint. Der Einfluß des Allgemeinbefindens auf die Störung zeigt sich auch deutlich darin, daß bei manchen Patienten, wie z. B. bei Fall 2, der über. haupt weniger geschädigt war als die andern, die Störung nur an schlechten Tagen in Erscheinung trat.

3. Es kommt nicht selten vor, daß außer dem einen , Ring", von dem wir bisher gesprochen haben, noch weitere auftreten: bei fortgesetzter Bewegung des Prüfungsobjektes gegen den Fixationspunkt wird das Objekt nochmals oder sogar mehrere Male für kurze Zeit undeutlich.

Die Zeit des Undeutlichwerdens ist aber gewöhnlich so kurz, daß die Patienten nicht immer imstande sind, alle die betreffenden Stellen 
genau anzugeben. Zuweilen aber gelingt dies, und dann summieren sich diese vielen Stellen verminderter Empfindlichkeit zu einem beinahe vollständigen zweiten , Ring" ${ }^{c}$, was bei dem einen untersuchten Patienten (Fall 5) sehr deutlich zutage trat. (Vgl. Fig. 11.)

Fall 5. P. M. Unteroffizier (Klavierbauer), 26 Jahre alt.

Am 13. X. 1916 durch Infanteriegeschoß verwundet.

Februar 1918. Auf dem linken Hinterhaupt $1 \mathrm{em}$ hinter dem. Ohransatz beginnend $8 \mathrm{~cm}$ lange Narbe, reaktionslos. Röntgenologisch: Mehrere Geschoßsplitter im Bereich der Wunde nachweisbar, die aber nur in den Weichteilen zu liegen scheinen (?). Rechtsseitige Taubheit, linksseitige hochgradige Sohwerhörigkeit. Nystagmus beiderseits, besonders nach links. Vorbeizeigen nach innen und unten links in der linken Hand, nach auBen in der rechten Hand.

Romberg ++ . Starke Gleichgewichts- und Gewichtsschätzungsstörungen. Ohrenärztlicher Befund: Beiderseitige Vestibulärläsion mit Kleinhirnbeteiligung. Pals langsam, labil.

Subjektive Beschwerden: Kopfschmerzen, besonders bei Witterungswechsel, Stechen, Ohrensausen, Flimmern, Schwindelgefühl, besonders beim Bücken. Blutandrang zum Kopf.

Sehschärfe: Beiderseits schwankend zwischen $5 / 6$ und beträchtlich weniger. Ophthalmologisch: o. B.

Dieser Kranke gab bei wiederholten Untersuchungen an, daß er an sehr vielen Stellen innerhalb eines Meridians eine verminderte Deutlichkeit des Prüfungsobjekts beobachtet habe, und verglich den Eindruck, den er dabei hatte, mit dem beim Durchschreiten eines hügeligen Geländes, wenn nämlich ein bestimmtes Gesichtsobjekt der Landschaft. abwechselnd sichtbar und wieder unsichtbar wird.

Auch das Auftreten zweier oder mehrerer , Ringe ${ }^{36}$ muß nach unserer Annahme von der Geschwindigkeit der Objektführung abhängen. Bleibt sie nämlich hinter der Geschwindigkeit zurück, mit der die Er. müdung zentralwärts fortschreitet, so muß es natürlich zu der gleichen Erscheinung des Verschwindens oder des Undeutlichwerdens des Prüfungsobjektes kommen, wie bei der ersten Registrierung.

Es ist nach alledem ohne weiteres klar, daß man je nach den Bedingungen, unter denen die Untersuchung stattfindet, ganz verschiedene Gesichtsfelder erhalten kann.

Unter den von uns gewonnenen Befunden verdient noch folgender Erwähnung: Wir haben schon gesagt, daß der Grad der Amblyopie nicht an allen Stellen des Ringes gleich groß ist. In dieser Beziehung zeigt sich eine gewisse RegelmäBigkeit darin, daB der , Ring" auf den temporalen Partien des Gesichtsfeldes bei manchen Patienten breiter und in viel stärkerem Maße amblyopisch ist, als auf den nasalen.

Zuweilen ist dieser Unterschied so groß, daß das Verschwinden bzw. Undeutlichwerden des Prüfungsobjektes überhaupt nur auf der temporalen Seite angegeben wird; auf der nasalen Seite ist das Undeut. lichwerden des Untersuchungsobjektes in solchen Fällen so gering und 
dauert nur so kurze Zeit, daß der Patient bei der mittleren Geschwindigkeit, mit der man das Prüfungsobjekt zu bewegen pflegt, gar nicht imstande ist, die Stellen verminderter Empfindlichkeit genau anzugeben. Erst nachträglich vermag der Kranke zu erklären, daß er das Prüfungsobjekt auch auf dieser (der nasalen) Seite manchmal nicht so deutlich gesehen hätte.

Unter solchen Umständen kann der Untersuchende natürlich nur auf den temporalen Seiten die Stellen verminderter Empfindlichkeit feststellen, was zur Folge haben muß, daß (auf dem Schema) die Ringform gar nicht zustande kommt, sondern nur auf den temporalen Seiten ein sichelförmiges ,Skotom" vorzuliegen scheint. Einen solchen Befund bei Fall 2 zeigen die Fig. $12 \mathrm{a}$ und $12 \mathrm{~b}$.

$\mathrm{Zu}$ einem ,Ringe ${ }^{60}$ kommt es in solchen Fällen nur dann, wenn man das Prüfungsobjekt beträchtlich langsamer als gewöhnlich bewegt und den Patienten nach jedem Meridian ausruhen läßt. Jetzt vermag der Kranke nämlich auch auf den nasalen Partien die Stellen verminderter Empfindlichkeit genauer anzugeben. Allerdings sieht dann der „Ring“" auf der nasalen Seite sehr schmal aus.

Auch dieser Befund bestätigt nur unsere Anschauung, daß es sich hier überall um Ermüdungseffekte handelt. Es ist eine oft betonte Tatsache, daß die den temporalen Gesichtsfeldpartien entsprechenden nasalen Netzhautregionen schneller und stärker ermüden als die anderen ${ }^{1}$ )

4. Zuletzt möchten wir noch über eine Beobachtung berichten, die namentlich in theoretischer Beziehung eine besondere Erwähnung und Beachtung verdient. Es handelt sich dabei um das Vorkommen halbseitiger (homonymer) Sehstörungen der beschriebenen Art (homonymer, halbringförmiger ,Skotome ${ }^{\text {" }}$ ).

Fall 6. F. M. Kaufmann, am 17. VII. 1918 durch ArtilleriegeschoB verwundet am Kopf und Rücken. Er klagte kurz nach der Verletzung über Sehstörungen auf der rechten Seite. Die Untersuchung ergab eine linksseitige Hemianopsie, Sehschärfe beiderseits $5 / \%$. Ophthalmologischer Befund normal, keine sonstigen Erseheinungen von seiten des Nervensystems. Die stereoskopische Röntgenanfnahme zeigt drei über stecknadelkopfgroße Fremdkörper in der Kopfschwarte des Hinterhauptes, einen Kleinen erbsengroßen Metallsplitter im Gehirn, im rechten Hinterhauptslappen.

Sehvermögen bessert sich in den nächsten Wochen. Pat. klagt jetzt über zeitweise Kopfschmerzen, leichte Ermüdbarkeit, besonders beim Sehen, es verschwimme ihm alles, und zwar ganz wesentlich auf der tinken Seite. Er habe leicht Hitzegefühl im Kopf, könne sich schlecht bücken. Sonst. Keine besonderen Klagen.

Da uns keine genaue Gesichtsfeldaufnahrae aus dem anfänglichen Krankheitsstadium des (zu uns erst verlegten) Patienten zur Verfügung

1) Vgl. z. B. Witbrand und Saenger, Über Sehstörungen bei fumktionellen Nervenleiden. Leipzig 1892. \$. 18/19, und $W$. König, Über Gesichtsfeldermüdung usw. Leipzig 1893, S. 6, 132 u. a. 
steht, so können wir natürlich nicht entscheiden, ob es sich bei der erwähnten hemianopischen Störung um eine völlige halbseitige Blindheit oder nur um eine Hemiamblyopie gehandelt hat. Der Kranke selbst glaubt zu wissen, daß er längere Zeit nach der Verletzung ,,auf der einen Seite gar nichts" gesehen hätte. Jedenfalls handelt es sich jetzt um eine Hemiamblyopie. Dieser Patient hatte nun, als er zu uns kam, in der linken Gesichtsfeldhälftte, aber auch nur hier, dieselbe Störung, wie wir sie bei den anderen Patienten im ganzen Gesichtsfeld festgestellt haben. Wir können sie hier wohl als den Ausdruck der einseitigen Hirnverletzung auffassen. Fig. 13a und 13b zeigen das Ergebnis einer von mehreren Aufnahmen, die wir gemacht haben ${ }^{1}$ ).

Auch bei diesem Patienten zeigte sich deutlich, daß das Gesichtsfeld bei jeder Neuaufnahme sich änderte: Der „Halbring“ lag bald mehr, bald weniger entfernt vom Fixationspunkt.

Man sieht ohne weiteres, daß die Ringe lediglich durch die beim Perimetrieren übliche Untersuchungstechnik entstehen. Der Untersuchende registriert die Stellen aufgehobener oder verminderter Empfindlichkeit in dem Augenblick, in dem der Patient das Versehwinden bzw. Undeutlichwerden des Objektes angibt. Nachträglich verbindet man die verschiedenen registrierten Punkte miteinander ,zum Gesichtsfeld", in der Meinung, daß während der weiteren Untersuchung die als normal registrierten Stellen in demselben Maße empfindlich geblieben seien, wie bei der Registrierung. Wenn aber, wie in unseren Fällen, der Zustand des Sehorgans, infolge abnormer Ermüdbarkeit, während der Untersuchung und infolge der Untersuchung sich dauernd verändert, dann ergibt die Verbindung der einzelnen registrierten Stellen ,zum Gesichtsfeld" ein völlig falsches, bizarres Bild. Daraus folgt, daß bei Patienten mit einer so abnorm raschen Ermüdung des Sehorgans das Ergebnis der gewöhnlichen Perimetrierung besonders bewertet werden muß; sonst kommt man zu der Annahme, daß es sich bei derartigen Patienten um wirkliche Skotome handelt, die aber in Wahrheit nur Scheinskotome sind ${ }^{2}$ ).

Möglicherweise könnte man mit anderen Methoden die Form und Ausdehnung des Gesichtsfeldes in einem bestimmten Stadium der Ermüdung festhalten. So z. B.

1) Wir könnten noch tüber eine ganze Reihe anderer, zum Teil ähnlicher Befunde berichten, worauf wir aber verzichten zu müssen glauben, da sie für das theoretische Verständnjs der Störung keine prinzipiell neuen Gesichtspunkte versehaffen.

2) Die Berechtigung, die einzelnen registrierten Stellen zu einem Gesichtsfeld zu verbinden, ist auch sonst, wenn keine so abnormen Verhältnisse vorliegen wie hier, nicht so ohne weiters selbstverständlich, wie man im allgemeinen anzunehmen geneigt ist. Diese für die theoretische Bewertung der Gesichtsfelduntersuchungen prinzipielle Frage soll bei einer anderen Gelegenheit erörtert werden. 
könnte man daran denken, an einem Halbhohlkugelperimeter, wenigstens in den Hauptmeridianen, an der Peripherie des Gesichtsfeldes verschiedene Prüfungsob. jelkte anzubringen und gewisserma Ben das ganze Gesichtsfeld simultan aufzunehmen, indem man den Patienten auffordert, anzugeben, wie viel Objekte or gleichzitig gesehen hat. Bei einer zweiten Prüfung kann man andere Meridiane wählen und die Untersuchung in ähnlicher Weise wiederholen usw. Selbstverständlich würde eine solche Gesichtsfeldaufnahme aus verschiedenen nabeliegenden Gründen kein Äquivalent bilden für das übliche Perimetrieren. Allein wir würden mit dieser Methode immerhin ein zutreffenderes Bild von der gerade vorhandenen Gesichtsfeldaus. dehnung erhalten als mit Hilfe der ublichen Methode.

Wenn man nun die von anderer Seite beschriebenen Ringskotome mit den von uns festgestellten vergleicht, so findet man sofort die große Übereinstimmung im Aussehen der registrierten Befunde, Wir finden z. B. bei Zade (a. a. O.) folgende Angaben: Bei einem Soldaten, der monatelang Dienst an einer Ballonabwehrkanone getan hat, ẹgab die Gesichtsfeldprüfung das Vorhandensein eines peripheren Ringskotoms an beiden Augen. Die von Zade zuerst - in Ermangelung eines Perimeters - campimetrisch, später auch an Perimetern mit Kreisbögen von verschiedenem Radius vorgenommenen Untersuchungen ergaben ähnliche Ringskotome bei einer sehr großen Zahl von Personen, deren Augen anhaltend intensivem Licht ausgesetzt waren (Mannschaften im Fliegerdienst und beim Telegraphenbau). Aus Zades Schilderung entnehmen wir folgendes: „Die Ringform ist ganz ausgesprochen; der Ring ist in einigen Fällen geschlossen, in anderen fehlt nur der obere Teil, und einigemal bestand das Skotom nur aus einer temporal gelegenen Sichel. Die Zone des ringförmigen Gesichtsfelddefektes ist nur einen oder einige Winkelgrade breit. Nur die Mitte der Ringzone ist ein absolutes Skotom; am Rande derselben, also ehe der zur Untersuchung benutzte Gegenstand ganz verschwindet, erscheint er schwächer. Diese Zone des relativen Skotoms ist so schmal, daß sie nur bei langsamem Perimetrieren zu erkennen ist". (Zade, Dies. Arch. 91, S. 159/60.)

Zade erwähnt auch, daß er hin und wieder mehrere konzentrisch zueinander gelegene Ringe gefunden hat, und $\mathrm{da} \beta$ bei wiederholten Prüfungen keine einheitlichen Ergebnisse zu erzielen waren. Die für unsere Befunde so charakteristische Tatsache, daß die Lage der Skotome bei einzelnen Untersuchungen verschieden ist, finden wir besonders erwähnt bei Inouye.

Eine Veränderung der Lage des Ringskotomes bei Veränderung der Versuchsbedingungen beobachtete auch Wölfflin (a. a. O.), denn nur so ist wohl seine Angabe zu verstehen, daß das Skotom bei einer "Campimeteruntersuchung wesentlich andere Grenzen aufweist als bei einfacher Perimetrierung".

Als Folge verschiedener Untersuchungsbedingungen ist wohl auch die Tatsache zu betrachten, daß verschiedene Autoren bei der gleichen 
Art der Schädigung die "Skotome" in versehiedenem Abstande vom Fixierpunkt fanden: Zade fand sie bei Mannschaften im Fliegerdienst regelmäßig ganz peripher, Doeschate vielfach nahe am Fixationspunkte, bis zu $25^{\circ}$.

Etwass Genaueres über die Veränderung der Skotome unter dem Einflusse der veränderten Untersuchungsbedingungen ist nach den vorliegenden Mitteilungen leider nicht auszusagen, weil es an daraufhin gerichteten Untersuchungen in allen Publikationen fehlt. Da aber gerade die Veränderlichkeit der Skotome sich uns von so wesentlicher Bedeutung für ihre Erklärung erwiesen hat, so ist man nicht in der Lage, über die Natur der in der Literatur mitgeteilten Störungen etwas absolut Sicheres auszusagen. Es scheint uns wegen wesentlicher Ubereinstimmung sehr nahe zu liegen, daß überall wohl dieselbe Erscheinung vorgelegen hat, daß es sich also auch bei den in der Literatur behandelten Fällen um ein Produkt der Untersuchungstechnik bei abnorm ermüdbaren Sehorganen gehandelt hat, mit dem einzigen Unterschied, daß wenigstens bei den Fällen einzelner Autoren (z. B. Zade, Doesschate) die Unterwertigkeit der Sehsubstanz durch eine Schädigung der Netzhaut oder der Sehnerven, bei unseren Fällen aber durch eine Schädigung zentraler Abschnitte verursacht wurde. Daher kommt es auch, daß Zade nur auf einem Auge das Vorkommen eines peripheren Ringskotoms beobachtet hat, nämlich bei Patienten, die meistens nur das eine Auge dem schädlichen Lichte anhaltend ausgesetzt hatten.

Da aber, wie gesagt, das in der Literatur vorliegende Material für eine sichere Beurteilung nicht ausreicht, so halten wir auch die Möglichkeit für nicht ausgeschlossen, daß in manchen von anderen Autoren beobachteten Fällen wirkliche Slkotome als Folgen umschriebener gröberer Netzhautschädigung vorgelegen haben. So scheint es sich z. B. bei dem ersten von Doesschate mitgeteilten Fall zu verhalten. Dafür scheint wenigstens $\mathrm{zu}$ sprechen, daB bei Kontrolluntersuchungen sich stets die gleichen Skotomgrenzen zeigten. Damit würde auch im Einklang stehen, daß der von Doesschate (Abb.1, S. 31 a. a. O.) wiedergegebene Gesichtsfeldbefund wesentliche Verschiedenheit aufweist gegenüber den eigentlichen Ringsskotomen, die dieser Autor (Abb. 2, S. 32 a. a. O.) bringt. Wahrscheinlich hat die erste Beobachtung mit den sog. Ringsskotomen nichts zu tun.

Nur in einzelnen Fällen sind die Tatsachen ausreichend, um sicher sagen zu können, daß es sich um dieselben Erscheinungen gehandelt hat wie bei uns, so z. B. bei dem Fall von Inouye und dem von Wölfflin. In beiden Fällen spielte keine periphere Schädigung für die Entstehung der Ringsskotome eine Rolle; bei dem Fall von Inouye war wie bei unseren Fällen eine Kopfverletzung die Ursache.

Die weitgehendste Utbereinstimmung mit unseren Befunden, daß 
nämlich beim Durchperimetrieren eines Meridianes das Prüfungsobjekt von Strecke zu Strecke verschwindet, dann nach längerer oder kürzerer Zeit wieder auftaucht, dann bald wieder versohwindet usw, stellt eine von Wilbrand bereits 1891 beobachtete Erscheinung dar, die er als „,oszillierendes Gesichtsfeld" bezeichnet hat (vgl. Text-Fig. 2).

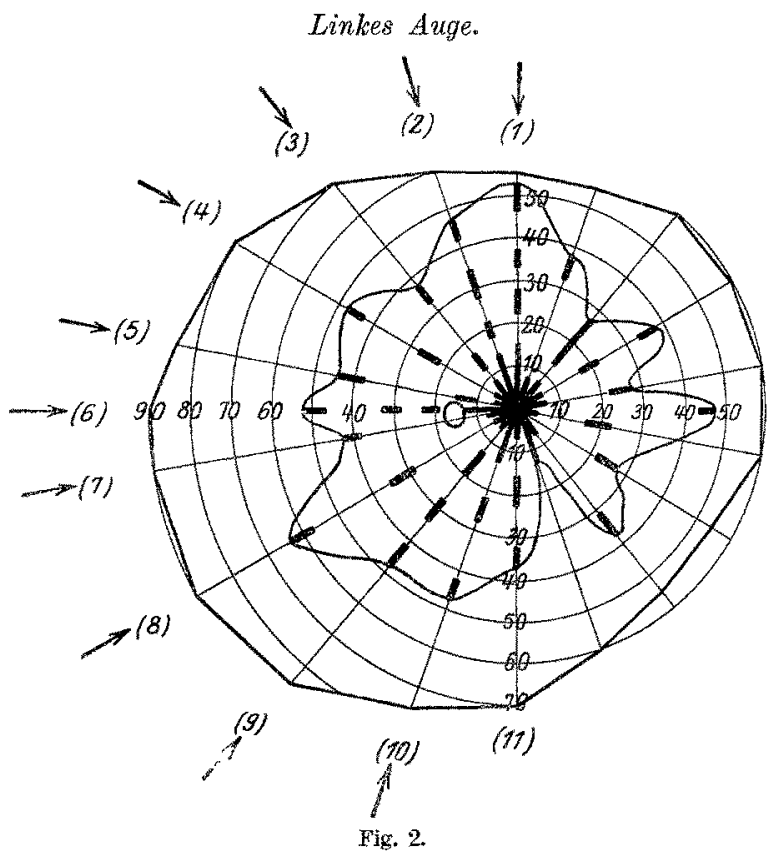

„Die schwarz gehaltenen Striche markieren die Strecken, auf welchen das Untersuchungsobjekt von $15 \mathrm{~mm}$ weiß wahrgenommen wurde, in den freigelassenen Zwischenräumen fehlte die Empfindung für den Augenblick der Untersuchung." (Wilbrand und Saenger, a. a. O. S. 20.)

Eine weitere Úbereinstimmung besteht darin, daß ein derartiges oszillierendes Gesichtsfeld, wie Wilbrand hervorhebt, fast bei jeder Untersuchung eine andere Form hat.

Auch die Erklärung Wilbrands stimmt insoweit mit der unsrigen überein, als auch Wilbrand die Ursache der Erscheinung in einer abnormen Ermüdbarkeit des Sehorgans (im Sinne Herings) sieht.

Freilich bedarf es noch einer näheren Erklärung, warum in den Wilbrand schen Fällen die abnorme Ermüdbarkeit nicht zu den typischen „Ringsskotomen ", sondern zu dem eigentümlichen oszillierenden Gesichtsfeld geführt hat. Da wir selbst Patienten mit solchen Gesichtsfeldern nicht untersuchen konnten, wollen wir diese Frage offen lassen.

Noch ein Wort zur Frage der Hysterie: Von einigen Autoren wurde das „Ringskotom" für eine hysterische Erscheinung erklärt (Inouye, 
Wölfflin). Um eine hysterische Erscheinung handelt es sich aber sicher nicht, sondern um Ermüdungserscheinungen, die zwar gegenüber den bei normalen Individuen bestehenden Verhältnissen abnorm gesteigert sind, die aber völlig den Gesetzmäßigkeiten der physiologischen Optik entsprechen.

Nie haben wir Ringskotome bei Hysterischen gefunden, sondern nur bei Kranken mit organischen Schädigungen des Sehapparates (immer handelte es sich, mit Ausnahme eines einzigen Falles, bei dem eine Schädigung der Chiasmagegend vorlag, um eine Schädigung der Sehsphäre), und zwar bei solchen, die gar keine hysterischen Komplikationen boten. Auch bei den in der Literatur mitgeteilten. Fällen ist die Annahme einer hysterischen Erscheinung überall, wo sie gemacht worden ist, nur aus Verlegenheit herangezogen worden, weil man die Gesetzmäßigkeit der Erscheinung nicht erkannte.

Die beschriebene Störung hat eine große praktische Bedeutung. Sie kommt den Kranken oft in der Form großer Flecke im Sehfeld zum Bewußtsein und beeinträchtigt sehr ihre Sehfähigkeit, besonders bei angestrengter Arbeit. Auch hierin besteht ein großer Gegensatz gegenüber den hysterischen Erscheinungen, die praktisch fast bedeutungslos zu sein pflegen.

\section{Bemerkungen zu Tafel $I$.}

Die auf der Tafel abgebildeten Gesichtsfelder gehören zu den einzelnen mitgeteilten Fällen, und zwar:

Fig. la-b (cf. S. 388), 5a-b (of. S. 391), 8 (ef. S. 395), 9 (ef. S. 395) zu Fall 1. Fig. 2 (cf. S. 389), 6a-6 (cf. S. 391), 12a-b (cf. S. 398) . . . , , 2 .

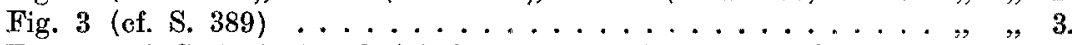
Fig. 4. (cf. S. 389), 7a-b (cf. S. 391), 10a-b (cf. S. 396) ..., ," 4.

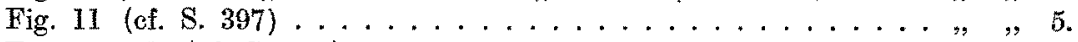

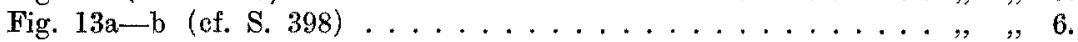

